\title{
Prophylactic strategies to control chikungunya virus infection
}

\author{
Friederike I. L. Hucke ${ }^{1} \cdot$ Malena Bestehorn-Willmann ${ }^{1} \cdot$ Joachim J. Bugert ${ }^{1}$ (i) \\ Received: 29 June 2020 / Accepted: 11 December 2020 / Published online: 15 February 2021 \\ (c) The Author(s), under exclusive licence to Springer Science+Business Media, LLC part of Springer Nature 2021
}

\begin{abstract}
Chikungunya virus (CHIKV) is a (re)emerging arbovirus and the causative agent of chikungunya fever. In recent years, CHIKV was responsible for a series of outbreaks, some of which had serious economic and public health impacts in the affected regions. So far, no CHIKV-specific antiviral therapy or vaccine has been approved. This review gives a brief summary on CHIKV epidemiology, spread, infection and diagnosis. It furthermore deals with the strategies against emerging diseases, drug development and the possibilities of testing antivirals against CHIKV in vitro and in vivo. With our review, we hope to provide the latest information on CHIKV, disease manifestation, as well as on the current state of CHIKV vaccine development and post-exposure therapy.
\end{abstract}

Keywords CHIKV epidemiology $\cdot$ Antiviral design $\cdot$ Antiviral testing $\cdot$ Vaccines $\cdot$ Monoclonal antibodies

\section{Introduction CHIKV}

\section{Taxonomy, structure and genome organisation}

Chikungunya virus (CHIKV) is an "old world" alphavirus (family: Togaviridae).

Alphaviruses are enveloped viruses with a diameter of about $70 \mathrm{~nm}$ and single-stranded positive sense RNA (+ ssRNA). CHIKV belongs to the Semliki Forest virus antigenic complex and is closely related to O'nyong-nyong virus [1]. Other viruses in this particular complex are Sempliki Forest virus, Ross River virus and Mayaro virus. Phylogenetic relationships of alphaviruses are shown in $1^{*}$ MERGEFORMAT Fig. 1. The virion is formed by an envelope consisting of a lipid bilayer and a lattice made up of 240 heterodimers of the viral envelope proteins E1 and E2 which are organised into 80 trimeric spikes. The E1 and E2 proteins are transmembrane glycoproteins and the $\mathrm{C}$-terminal domain of the $\mathrm{E} 2$ protein has direct contact with the virus's nucleocapsid (NC) core [2]. Thus, the virion's outer protein

Edited by Detlev H. Kruger.

Joachim J. Bugert

Joachim1Bugert@bundeswehr.org

1 Bundeswehr Institute of Microbiology, Neuherbergstraße 11, 80937 München, Germany shell is tightly associated with the 240 capsid proteins (Cp) that build up the icosahedral nucleocapsid (NC).

The CHIKV genome consists of about 11,800 nucleotides and follows the general organisation of all alphaviruses. It contains two open reading frames (ORFs) which encode the non-structural (ns) or replicase polyprotein and the structural polyprotein [6]. The RNA has a 5`7-methylguanosine cap and a 3 poly-A tail and, thus, mimics the structure of cellular mRNA [7]. Two-thirds of the 5'-ORF encode for four essential non-structural proteins (nsP1-4) which are required for virus replication and constitute the RNA replicase. The nsPs interact with cellular factors and form the replication complexes (RCs) which are responsible for the synthesis of the double-stranded (ds)RNA replicative intermediates. These dsRNAs are the templates for the positive strand viral (42S) genomic and (26S) subgenomic RNAs. The subgenomic RNA thus constitute the last third of the viral RNA and is translated into the structural proteins (capsid (Cp), E3, E2, $6 \mathrm{~K} / \mathrm{TF}$, and E1) $[6,8]$. For a brief characterisation, see $\perp^{*}$ MERGEFORMAT Table 1.

For a more detailed description especially with regard to the fact that the nsPs pose possible targets for antiviral drugs we refer the reviews of Strauss and Strauss [6], Silva and Dermody [8], and Pietila, Hellstrom [9] on the subject of alphavirus and CHIKV structure, replication and life cycle. 


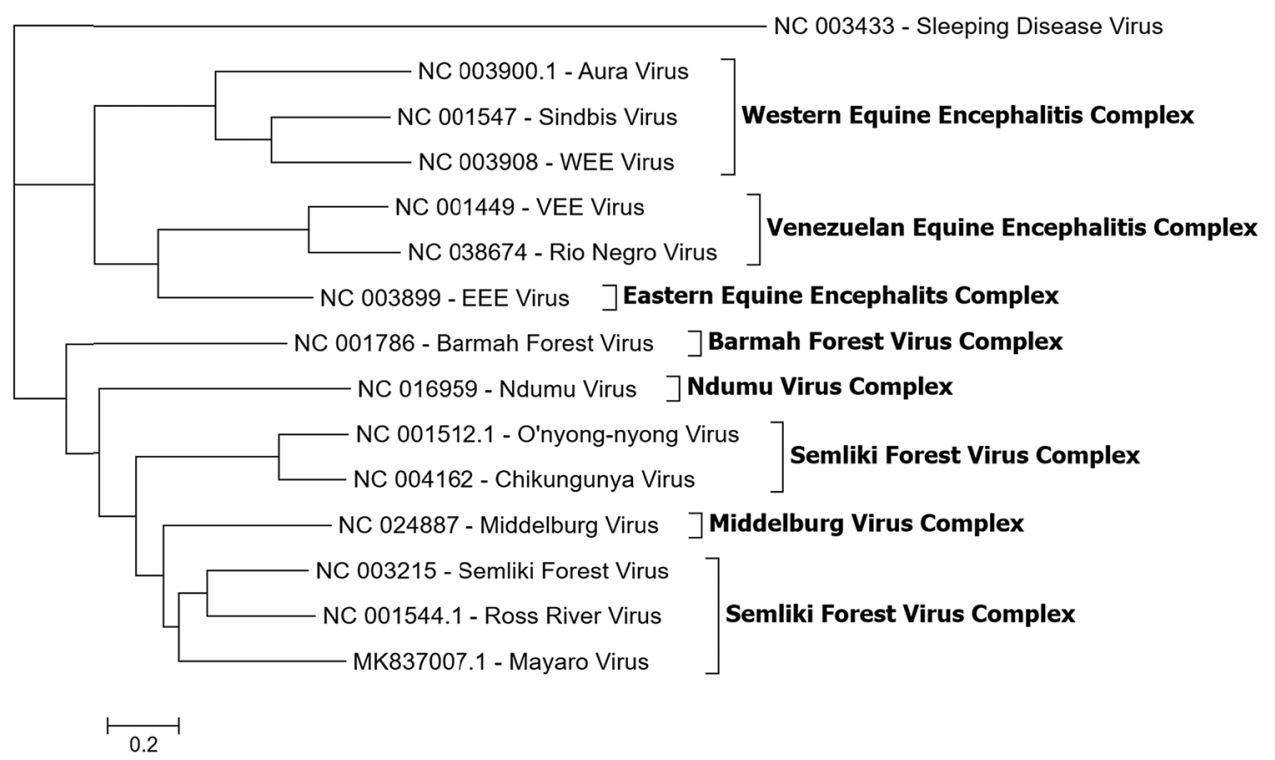

Fig. 1 Molecular phylogeny of the medically most relevant and representative alphaviruses. CHIKV belongs to the Semliki Forest virus antigenic complex and is closely related to O'nyong-nyong virus [3]. The phylogenetic tree was inferred based on a MAFFT-E translational alignment. Fifteen representative nucleotide sequences of the ORF 2 encoding the structural proteins of alphaviruses were used.
A maximum likelihood phylogenetic tree was generated using the $\mathrm{GTR}+\mathrm{F}+\mathrm{I}+\mathrm{G} 4$ model and 1000 bootstrap replicates. The software used was IQ-TREE $[4,5]$. Virus names are shown next to the GenBank accession numbers. EEE Eastern equine encephalitis; VEE Venezuelan equine encephalitis, WEE Western equine encephalitis

Table 1 Functions of the various structural and non-structural Proteins of CHIKV

\begin{tabular}{|c|c|c|}
\hline Protein & $\operatorname{Size}(\mathrm{aa})$ & Function \\
\hline Non-structural Proteins & 2474 & $\mathrm{P} 1234$ precursor protein \\
\hline nsP1 & 535 & $\begin{array}{l}\text { Methyltransferase and guanylyl transferase activities capping and methylation of new viral RNAs; is the } \\
\text { only membrane anchor for the replication complex }\end{array}$ \\
\hline nsP2 & 798 & $\begin{array}{l}\text { C-terminal cysteine (auto)protease activity cleaves initial polyprotein into individual non-structural pro- } \\
\text { teins thus enabling viral replication; has also N-terminal helicase, nucleoside triphosphatase and RNA } \\
\text { triphosphatase activities; interferes with the host cells mRNA transcription \& translation and inhibits } \\
\text { interferon signalling }\end{array}$ \\
\hline nsP3 & 530 & $\begin{array}{l}\text { Interacts with several host proteins, possibly modulates protein poly- and mono-ADP-ribosylation; influ- } \\
\text { ences host cells stress response; Phosphoprotein, important for RNA minus-strand synthesis }\end{array}$ \\
\hline $\mathrm{nsP} 4$ & 611 & $\begin{array}{l}\text { RNA-dependent RNA polymerase (RdRp) essential for viral replication; presumed terminal transferase } \\
\text { activity }\end{array}$ \\
\hline Structural Proteins & 1244 & Structural precursor protein \\
\hline C (Capsid) & 261 & $\begin{array}{l}\text { Forms nucleocapsid core by encapsidating genomic RNA; carboxyl domain is an autocatalytic serine } \\
\text { protease }\end{array}$ \\
\hline P62/PE2 & 487 & Precursor/Intermediate, later cleaved into E2 and E3 by host furin protease \\
\hline E1 & 435 & $\begin{array}{l}\text { Surface protein; Type II fusion protein; mediates fusion of viral envelope and cellular membrane by fusion } \\
\text { peptides }\end{array}$ \\
\hline E2 & 423 & $\begin{array}{l}\text { Surface protein; major target of neutralizing antibodies; mediates binding to receptors and attachment fac- } \\
\text { tors on host cell membrane }\end{array}$ \\
\hline E3 & 64 & $\begin{array}{l}\text { N-terminal domain is uncleaved leader peptide of E2, suspected to shield fusion peptide in E1 during } \\
\text { egress }\end{array}$ \\
\hline $6 \mathrm{~K}$ & 61 & Leader peptide of E1; presumed ion channel; may enhance particle assembly and release \\
\hline $\mathrm{TF}$ & 76 & $\begin{array}{l}\text { Transframe protein, produced via ribosomal frameshifting, presumed ion channel, may enhance particle } \\
\text { release, shares N-terminus with } 6 \mathrm{~K}\end{array}$ \\
\hline
\end{tabular}

$A a$ amino acid number; $C$ capsid; $n s P$ non-structural protein; $R d R p$ RNA-dependent RNA polymerase; $T F$ transframe protein 


\section{Ecology and epidemiology}

Chikungunya virus (CHIKV) is an arthropod borne (arbo-) virus of the Alphavirus genus. It was first described in 1955 by Robinson and Lumsden after an outbreak in present day Tanzania in 1952. The word "chikungunya" is derived from the Makonde word "kungunyala" which means "that which bends up", thus describing the stooped bearing and rigid gait of infected individuals [10]. Since symptoms are very similar to Dengue fever, it is possible that retrospectively, reports of outbreaks could also be attributed to CHIKV reaching possibly even back until 1658 [11].

CHIKV is usually transmitted to humans by infected mosquitos from the Aedes genus, mainly Aedes aegypti, Aedes albopictus and Aedes polynesiensis, but Culex spp., Anopheles spp., and Mansonia spp. have been found infected with CHIKV as well [12]. CHIKV causes sporadic, more or less periodical outbreaks especially during rainy seasons when mosquito populations are high [13]. The virus can affect both arthropods and vertebrates, with the arthropod staying infected for all their life. CHIKV circulates in a sylvatic/ enzootic and in an urban cycle. In Africa, the sylvatic cycle is upheld by forest dwelling mosquitoes like Ae. furcifer, Ae. taylori, Ae. africanus and Ae. neoafricanus that infect vertebrates such as monkeys, rodents, and birds. Especially monkeys seem to serve as reservoir and amplification hosts in between epidemics [14].

So far, a sylvatic cycle has not been found in Asia, where the first CHIKV outbreak was reported in 1958. It is assumed that CHIKV maintains its presence by the urban cycle in this region [15]. Yet, the existence of a sylvatic cycle cannot be ruled out, since CHIKV-specific antibodies were found in Asian non-human primates (NHP) [16]. Furthermore, Mavale, Parashar [17] demonstrated that CHIKV-infected male mosquitoes can transmit the virus efficiently to females thus showing that a venereal (and probably a transovarial) transmission of the virus in the arthropods plays a role for the persistence of CHIKV in this particular region. Acharya, Paul [18] showed that in vitro mosquito cell-generated CHIKV has a lower infectivity in cell culture and caused less severe disease in mice compared to mammalian cell-generated CHIKV. This is due to the loss of glycosaminoglycan receptor binding of CHIKV on mammalian cell surface after the mosquito cell passage [18]. This suggests that transmission of CHIKV amongst the arthropods actually keeps infectivity of the virus downregulated.

Historically, CHIKV was mainly distributed in tropical and subtropical regions of sub-Sahara Africa and Southeast Asia causing sporadic outbreaks. CHIKV was, however, put into focus after a massive outbreak in Kenya in 2004 with close to half a million infected people. This epidemic initiated the spread to more than 22 countries and distributed the virus into regions with moderate climate (Fig. 2) [19]. In India, an outbreak of CHIKV affected more than 1.4 million people in 2005 and was followed by additional epidemics in 2006 and 2007 [20]. Originating in Africa, the 2004 outbreak expanded to the Indian Ocean, India, and Southeast Asia. CHIKV eventually reached Europe in 2007 leading to 205 confirmed cases of CHIKF in Castiglione di Ravenna in Italy [21]. Unlike the previous sporadic outbreaks, the 2004-2010 epidemic displayed autochthonous cases in temperate climates such as in Montpellier, France [22]. In 2013, CHIKV emerged in the Americas, firstly in the Caribbean Islands and reaching the South American continent in 2014 [23]. This led to increasing CHIKF cases and between 2014 and the end of September 2018, a total of 697,564 CHIKV cases have been notified in Brazil (including 94,672 laboratory-confirmed cases). The majority of new CHIKV cases in this year (January until 17. July 2020) have been reported from Brazil, Yemen, Bolivia and Thailand [24].

Until 2004, it has been widely accepted that the Ae. aegypti mosquito was the main urban vector of CHIKV [1].
Fig. 2 CHIKV distribution. World map displaying countries where autochthonous (locally initiated) chains of CHIKV transmission have been identified in the past. *In the continental USA, only travelassociated CHIKV infections have been reported in the past three years (2017-March 2020)

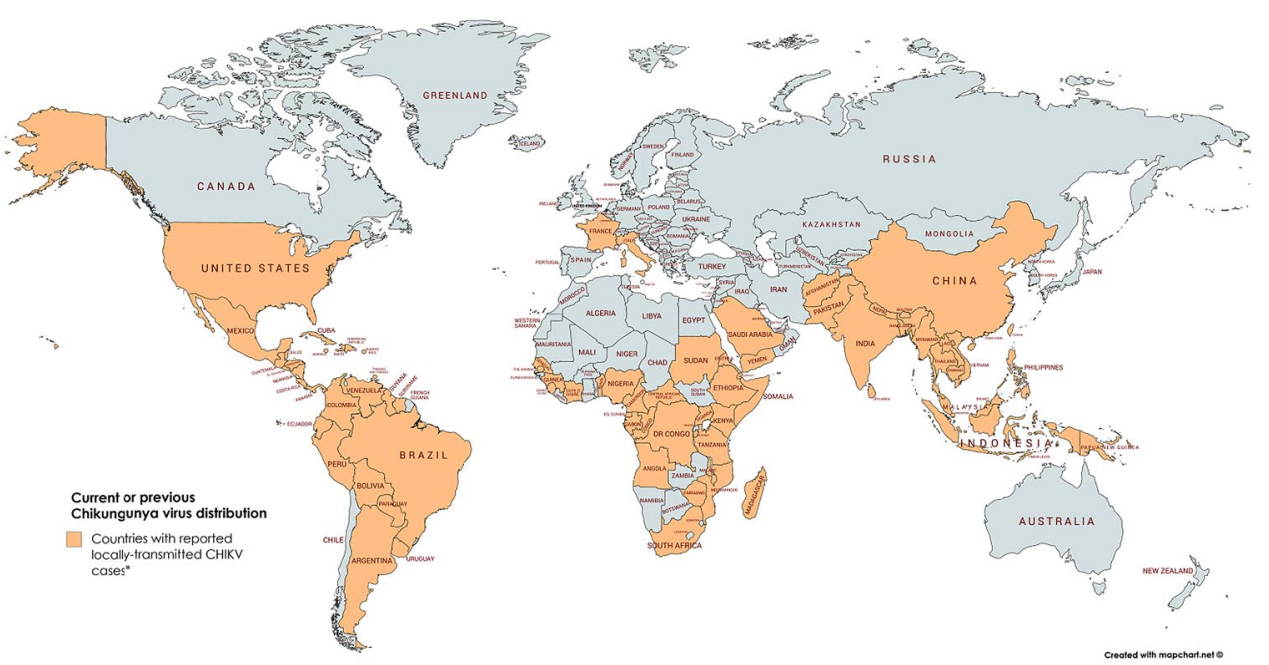


This arthropod is well adapted to the urban environment and can multiply fast in a short time. However, during the 2005/2006 outbreak on La Réunion Island the Asian "tiger mosquito" (Ae. albopictus) got into focus as far as CHIKV transmission was concerned [25]. With 4-8 weeks, Ae. albopictus has a long lifespan (depending on the temperature) and a flight radius of 400-600 m. Both, Ae. aegypti and Ae. albopictus are diurnal. Yet, the geographical distribution of Ae. albopictus is broader than that of Ae. aegypti, since it can exist in more temperate zones [26]. Eggs of Ae. albopictus are more resistant to drying-up and can stay viable throughout dry seasons. The mosquito recently expanded from Southeast Asia to Madagascar, most islands in the Indian Ocean, Africa, and the Americas. Due to climate change, it even made the transition to the temperate zones of Southern Europe (Italy, France).

\section{Phylogenetic studies and lineages}

Before the La Réunion outbreak, phylogenetic analyses based on both partial (E1 glycoprotein) and complete genome analyses revealed the existence of three distinct CHIKV phylogroups (strains) commonly referred to as the West African (WA), East-Central-South African (ECSA) and Asian genotypes [1]. Genome analysis of CHIKV strains isolated during the La Réunion epidemic in 2005 and 2006 revealed that the outbreak was caused by a mutated strain originating from the ECSA isolates [13]. This new mutant was then referred to as the Indian Ocean Lineage (IOL). When CHIKV was introduced into the Caribbean in 2013 it formed another sub-lineage within the Asian lineage (Asian/ American) [27]. As far as health issues are concerned, the most relevant lineages are the Asian (including the Asian/ American), the IOL sub-lineage and some ECSA strains. Multiple CHIKV outbreaks in the past 15 years go back to these three clades [28].

The question why CHIKV spread over the Indian Ocean and into areas with temperate climate was partly answered by Schuffeneckers discovery of a mutation (referred to as E1-A226V) at residue 226 of the membrane fusion glycoprotein E1. This mutation made CHIKV more adaptable to Ae. albopictus, as a study by Tsetsarkin and colleagues could demonstrate $[13,29]$. Additionally, reverse genetics identified possible mutations in the E2 glycoprotein (E2-G60D and E2-I211T) which were important for determining CHIKV infectivity in Ae. albopictus. Tsetsarkin et al., suspects a strong synergistic effect of the E2-G60D and E2-I211T mutations on CHIKV infectivity for Ae. albopictus, when expressed in combination with valine at position E1-226 [30]. In a follow-up study conducted in India another novel E2 mutation, L210Q, was present in all human and mosquito CHIKV isolates collected during 2009 [31]. This substitution was within the region of the E2 protein (amino acids E2 200-220) that determines mosquito cell infectivity for several alphaviruses [32-34].The virus' mutations turned Ae. albopictus from a secondary vector to the main transmitter of CHIKV. This enabled the new IOL to spread into regions where this arthropod is distributed. The mutation in the E1 protein had been observed before in Semliki Forest virus (SFV), another alphavirus, where the mutation made SFV less dependent on cholesterol for growth [35]. The efficiency of alphavirus' entry generally depends on the composition of the host cell membrane. Cellular membrane cholesterol is needed for membrane fusion and an efficient exit of progeny virus from infected cells. A mutation that made the virus more independent from cholesterol content in membranes would improve its fitness, especially in insects which have a different lipid composition in the cells. Experimental infection of Ae. albopictus with the non-mutated CHIKV strains actually proved that these lineages were not able to replicate as sufficiently in the tiger mosquito as the IOL strain and that the mutated sub-lineage had a significant increase in infectivity, dissemination and transmission by Ae. albopictus [29].

\section{Pathogenesis}

CHIKV is naturally transmitted to humans through the saliva of infected mosquitoes when they take a blood meal. Additionally, cases of mother to child transmission are known. Once the virus enters the skin, dermal fibroblasts seem to be the main site of viral replication and amplification [36, 37]. Proteins from mosquito saliva promote viral replication by counteracting the induction of antiviral genes, thus hampering the hosts' immune response [37]. Apart from dermal skin fibroblasts, skin keratinocyte and melanocytes are permissive for CHIKV infection as well [38]. Studies with NHP characterised the route CHIKV takes to reach other anatomical regions [39]. At the primary site of infection (skin) CHIKV infects macrophages. Viral particles are captured by dendritic cells (DCs) which transport the virus to the closest lymph nodes. Within the lymph node, viral particles are transferred to monocytes and macrophages which enter the bloodstream. After CHIKV reaches the lymph node, viremia sets in by the active infection of human blood monocytes and other peripheral blood mononuclear cells [40].

Via blood stream, CHIKV reaches the muscles and joints where the infection causes the main symptoms of CHIKFmyalgia and arthralgia. In the muscle, satellite cells seem to be the target for CHIKV, but primary human myoblasts are permissive to the virus infection as well and CHIKV could also be detected in skeletal muscle fibroblasts [41, 42]. CHIKV RNA and proteins could be found in the synovial tissues and fluids during acute and persistent arthralgia and various studies showed that synovial fibroblasts as well as macrophages are susceptible to CHIKV [12, 42]. 
In CHIKV-infected individuals, cartilage degradation and bone loss take place in the infected joints [43]. CHIKV also replicates and persists in osteoblasts [38].

CHIKV can target a wide range of secondary organs which may lead to severe complications in patients (i.e. renal, respiratory, hepatic, cardiac, and neural syndromes) [12]. CHIKV disseminates into the liver, the spleen, the heart, the kidneys and possibly the lungs [36, 39, 44]. Although CHIKV has not been considered a true neurotropic virus, it can cause neurological complications (e.g. meningitis, encephalitis, febrile seizures, Guillain Barré syndrome, neuro-ocular diseases) especially in the elderly and the very young [45]. In the Indian Ocean outbreak of 2005, a growing number of neurological symptoms associated with CHIKV were observed. Since then the tropism of this agent for the nervous system has been characterised better [46]. In patients displaying severe neurological symptoms, CHIKV could either be isolated from or detected via RT-PCR in the cerebrospinal fluid [45]. In vitro experiments showed, that CHIKV is able to infect and replicate in neurons, astrocytes, oligodendrocytes and microglia cells [47]. Despite these findings a consensus to the discussion whether the virus affects the nervous system directly or indirectly via a triggered immune-mediated effect has not been reached yet.

Inglis and colleagues concluded that a disrupted glia-neuron signalling could be a major driving factor in the development of CHIKV-associated neuropathology [48], a finding that was also confirmed in mice [49]. For a more detailed understanding of the current knowledge on pathogenesis and tissue tropism of CHIKV as well as on vector and animal host interactions we refer to the review of Matusali et al. [12].

\section{Clinical manifestation}

CHIKV causes a febrile disease called Chikungunya fever (CHIKF). Typically, patients develop symptoms after an incubation period of 1-12 days. High fever accompanied with headache, myalgia and joint pain are typical, in some cases haemorrhage and maculopapular rash may occur [8]. Especially musculoskeletal symptoms like persistent disabling polyarthralgia are a hallmark of the disease and have repeatedly been observed to go into a chronic state that may last months or even years in up to $50 \%$ of the patients [50]. A follow-up study done by Manimunda, Vijayachari [51] during an epidemic in Karnataka state, India in 2008, revealed that the arthritis caused by CHIKV is a chronic inflammatory erosive arthritis. Interestingly, the most common symptoms in this study were joint pain (98\%), fever (85\%), swelling around joints (53\%), rash (50\%), fatigue (49\%) and headache (38\%). After 9 months, $51 \%$ of the patients had been cured, leaving $49 \%$ in a chronic state of the disease [51].
If an immunologically naïve population is confronted with CHIKV, the morbidity ranges from $34 \%$ (like in La Réunion in 2005) to $63 \%[52,53]$.

Although CHIKV is often self-limiting and has a low mortality rate $(0.1 \%)$ [54], complications may arise especially in the elderly and the young. The major outbreaks between 2005 and 2016 recorded a significant number of patients suffering from serious complications such as cardiovascular disorders, involvement of the central nervous system (CNS), respiratory failure, pre-renal failure, and severe acute hepatitis. The mortality rate among these severe cases ranged from 10.6 to $35 \%$ with most patients dying of heart failure, multiple organ failure syndrome or toxic hepatitis $[13,52]$.

Patients under 1 year or over 65 years of age, have a higher risk of being affected by a CHIKF associated CNS disease (including encephalitis). Among the most frequently diagnosed CHIKV-associated neurological complications are encephalitis, optic neuropathy, neuroretinitis and Guillain-Barré-Syndrome, but also occasionally meningoencephalitis, myelitis and polyradiculitis [46]. In a cohort study during the CHIKV outbreak on La Réunion between 2005 and 2009, Gérardin (2016) determined the case fatality rate of CHIKV-associated encephalitis to $16.6 \%$ among neonates and the proportion of children discharged with persistent disabilities (seizures, cerebral palsy) was estimated between 30 and $45 \%$. Various cases were CHIKV has been transmitted vertically from mother to neonate are known. This poses the risk of neurodevelopmental delays, cerebral palsies and microcephaly in the infant [55].

\section{Differential diagnosis, CHIKV diagnostics, and surveillance}

The initial signs of CHIKV disease (fever with or without headache and/or arthralgia) are also common in several other diseases. Depending on the patient's history (place of residence, travel history, and exposure), different diseases can be considered in the differential diagnoses such as Malaria, Dengue (DENV), Leptospirosis, and other alphavirus infections like Mayaro, Ross River, O'nyong nyong and Sindbis [56]. Apart from being mistaken for another disease, CHIKV infection often go hand in hand with Malaria, Dengue (DENV) or Zika virus (ZIKV) infection and the four diseases share a common set of initial symptoms (headache, fatigue, and myalgia/arthralgia). It is very important for the patient to have quick and reliable diagnosis because prognosis and patient care differ for these diseases [57]. Especially Malaria and Dengue have the potential for much worse outcomes including death and it is thus eminent to distinguish the pathogens. So far, CHIKF can only be distinguished from Dengue fever by 
virus testing and early diagnoses might prevent complications in the patient like haemorrhages, acute respiratory distress syndrome, renal failure and arthritis [58].

Acute CHIKV infections are diagnosed either by virus isolation in cell culture, detecting virus genome or serologically, by detecting specific IgM antibodies [59]. For more detailed information on how to test during what phase of the disease we refer to Barrera, Hunsperger [56]. There are commercial serological assays (enzyme-linked immunosorbent assay (ELISA), immunofluorescence assay (IFA)) and molecular (RT-PCR) detection systems available $[60,61]$.

Various studies have been conducted in order to find the best diagnostic method. PCR methods seemed to outperform serological assays, which were less sensitive and specific, as they depend to a great extent on the skills of the performing laboratories [59]. Furthermore, due to crossreactivity of antibodies to common antigens among CHIKV related alphaviruses (e.g. Barmah virus, Ross River virus and Mayaro virus (MAYV)), false positive and false negative results are possible and serologic diagnosis remains a challenge [62]. This is particularly difficult when the viruses have the same geographical distribution and share a set of common symptoms as is the case with CHIKV and MAYV which both are endemic in Brazil and its neighbouring countries [63].

Questionable results should be confirmed with a second assay, e.g. serum virus neutralisation assay (VNT), which is a confirmatory test and considered more specific than ELISA and IFA tests [64].

Even before CHIKV was (re)introduced into the Americas, the Centres for Disease Control and Prevention (CDC) together with the Pan American Health Organization (PAHO) published the guide for 'Preparedness and Response for Chikungunya Virus Introduction in the Americas' [56]. However, although recommendations for surveillance had been provided, some American countries had not established a surveillance system for this particular disease due to lack of clinical and public awareness as well as non-existence of laboratory capacity for testing [65]. According to a retrospective study on Puerto Rico, passive case surveillance seemed most practical and feasible approach to compare epidemiologic trends across regions affected by chikungunya and other emerging infectious diseases [65]. Passive surveillance for a disease happens when a sick person seeks medical care, a doctor suspects a certain pathogen as the causative agent, and the case is reported to public health authorities either before or after laboratory diagnostic testing is done. Passive surveillance is particularly useful to monitor epidemiologic trends in diseases [65]. Yet, the infrastructure for passive case surveillance system might still have to be established, which proves difficult in developing countries with lack of resources. In Puerto Rico, the already existing passive dengue surveillance system that existed since the 1960s was modified to fit the new agent CHIKV [65].

If a disease is already endemic in a country, surveillance of this specific disease is only justified if appropriate actions to ban it are planned [66]. As there is no specific treatment for CHIKF available, the clinical outcome of a patient is only influenced by early detection when a more severe disease can be excluded. In tropical areas where vector activity is to be expected all year long, the same control measures as for DENV might help avoid high infection numbers and a ready detection of a new outbreak could help reduce the burden to public health by raising awareness [66]. Between epidemics, serological surveys are hardly done. Yet some studies for active surveillance have been conducted According to a study that investigated the cause of febrile illness in children during an inter-epidemic period in 5 Asian countries, CHIKV was responsible for $35 \%$ of all fevers [67]. Other seroprevalence studies in Africa confirmed that CHIKV was circulating endemically [66]. There was a program from the Pacific Public Health Surveillance Network for specific surveillance strategies of CHIKV, DENV and other febrile diseases including case detection and management, laboratory confirmation, vector control and raising awareness [68]. However, their website has not been updated since May 2016, so no data can be found on the current state of CHIKV surveillance in this area [69].

In Europe and non-endemic countries, CHIKV cases may occur either travel associated or if a viremic traveller enters areas where Ae. albopictus is present and finds suitable environmental conditions for the transmission of the virus (which is the case when daily temperature are $\geq 18^{\circ} \mathrm{C}$ ) [70]. Thus, travellers returning from endemic areas with febrile illness should be readily tested for Malaria, DENV and CHIKV [66]. According to the European Centre for Disease Prevention and Control (ECDC) the risk assessment states the chance for CHIKV infection in France and Italy, where autochthonous cases have been repeatedly reported, as being moderate [71]. Public awareness should be raised as an essential part of any DENV/CHIKV control program to help identify potential cases and start entomological investigations and possible vector control measures to curb further spread [66].

\section{Strategies against emerging infectious diseases}

The following section will give information on drug development and evaluation as well as on vaccines that are currently being investigated in clinical trials against CHIKV. For those interested in current antivirals against alphaviruses in general and other agents relevant in medical biodefence, we recommend the review of Bugert et al. [72]. 


\section{Drug development}

There are three major approaches to finding new drugs: (1) in traditional or phenotypic drug discovery, also called forward pharmacology, potential drugs are screened based on measures of phenotype. (2) Rational drug design, which is also referred to as reverse pharmacology, is based on the knowledge of a biological target which may function at critical intervention points in a disease process and might be blocked by drugs. (3) Repurposing already existing drugs is also a commonly used approach. We will not detail the different approaches but recommend the reviews of Pathel [73], Takenaka [74] and Rester [75].

\section{Methods for in vitro and in vivo evaluation of antiviral compounds}

\section{Viruses}

Bio-safe surrogates for CHIKV help avoiding the need for BSL-3 facilities. The BHK replicon cell line for example contains a persistently replicating CHIKV replicon [76]. To study virus entry and possible effects of agents on this particular step of CHIKV life cycle requires other models such as CHIKV pseudo-particles that carry the envelope proteins and are tagged with a luciferase reporter [77, 78]. Lucas-Hourani, Lupan [79] screened for CHIKV nsP2 protease inhibitors without using life virus infection. He transfected human HEK-293 T cells with various plasmids that encoded for the sequence of CHIKV nsP2 protein, a luciferase reporter gene and two transcription factors.

Some research groups use the vaccine strain 181/Clone 25 as a model virus. This is a live-attenuated derivative of Southeast Asian human isolate strain AF15561 (GenBank accession no. L37661, listed as TSI-GSD-218). It displays reduced virulence but still has the complete virus' life cycle [80, 81]. Gorchakov, Wang [82] revealed 10 nucleotide differences in the genome compared to its parental strain. Only 5 mutations actually caused an amino acid substitution, the rest were silent mutations. This strain could easily re-evolve back into a more infectious strain [82].

Wild-type (WT) strains with a complete life cycle include clinical isolates like the DRDE-06 strain (GenBank: EF210157) [83], DMERI09/08 strain [84], or laboratory CHIKV strains such as ROSS [84, 85], LR2006_OPY1 (GenBank: DQ443544.2) and the Indian Ocean strain 899 (GenBank: FJ959103.1) [86].

Reporter viruses for high-throughput screening, include recombinant CHIKV expressing GFP (e.g. CHIKV-118GFP) [87] or luciferase genes [76].

For the initial identification and screening of molecules with antiviral activity, cell viability/cytopathogenic effect (CPE) reduction assays are usually employed. These assays also allow the evaluation of the cytotoxic effect of putative antiviral molecules on the cells. African green monkey kidney cells (Vero cell line) are the most commonly used cells in these assays with CHIKV. Other cell types used for CHIKV antiviral screening include baby hamster kidney (BHK) cells, human foetal lung fibroblast (MRC-5) cells, bronchial epithelial cells, human embryonic kidney 293 (HEK-293) cells and human hepatocarcinoma (Huh-7) cells ([86-90]. Nevertheless, the fact that these commonly used cell lines have little clinical relevance in CHIKV disease is a big disadvantage. Some groups used human muscle satellite cells [41]. Labadie, Larcher [39] found out that macrophages are the main cellular reservoirs during the late stages of CHIKV infection in NHP. Teng, Foo [91] showed that monocytes are the major PBMC (primary blood mononuclear cells) subset targeted by CHIKV in the blood. These cells may be more relevant cell lines, especially as they are suspected to contribute to the long-term effects of CHIKV in humans. Yet, since they are a primary cell lines which are not immortalised, they are not well suited for high-throughput screening (HTS). Furthermore, human dermal fibroblasts seem to be the initial target cells in CHIKV infection, as they are the first to be infected after a mosquito bite [36, 42]. Abdelnabi and colleagues successfully used the human skin fibroblast cell line CRL-2522 as a model for testing CHIKV antivirals [92].

The effect of CHIKV on the cells (CPE) can be either evaluated microscopically and/or quantitatively measured by colorimetric assays e.g. resazurin fluorescence reduction assay [90], the MTS/PMS method [86] or neutral red dye uptake [83]. Additionally, in assays with adherent cell lines, xCELLigence real-time cell analysis (RTCA) screening is an accurate method to investigate the properties of antiviral compounds in infected and non-infected cells, [93]. This method is an established electronic cell sensor array using impedance measurement to detect the number of adhered (and thus viable) cells. It has the big advantage of measuring the cell viability continuously, whereas the aforementioned colorimetric assays are endpoint assays and do not provide information on the initiation of CPE or the changes in reaction rate of the virus propagation over time.

\section{Animal models}

There is a variety of mouse models to study CHIKV pathogenesis. Adult immunodeficient mice such as AG129 are being used for lethal infection models [86]. Pal, Dowd [94] used Ifnar ${ }^{-/-}$mice to assess the antiviral efficacy of small molecules and monoclonal antibodies against CHIKVinduced death. To study the efficacy of drug therapy against arthritis and inflammation caused by CHIKV, non-lethal infection models with immunocompetent mice such as C57BL/6 and Swiss albino mice are being employed [77, 
95]. Other research groups use cynomolgus macaques (Macaca fascicularis) or rhesus macaques (Macaca mulatta) to study CHIKV, since these NHP show similar signs of CHIKF as humans, including fever and skin rashes [96]. In his study with immunocompetent macaques, Labadie showed that long-term CHIKV infection was observed in joints, muscles, lymphoid organs, and liver, and that this fact might contribute to the long-term symptoms in humans. Yet, severe signs such as arthritis, meningoencephalitis and death were only observed after infecting the macaques with higher doses of CHIKV [39].

\section{Current strategies against CHIKV}

Although mortality rates of CHIKF are rather low with $0.1 \%$, the impact of the diseases on the patient is severe, especially when the virus hits a naïve population. The epidemic on La Réunion in 2005-2006 displayed CHIKF with atypical symptoms going hand in hand with severe morbidity (34.4\% of affected patients) and a higher mortality rate $(10.6 \%)$ [52] while affecting nearly $34 \%$ of the overall population of the island [19]. Apart from the suffering which patients have to endure personally, the economic and social impact of the disease seriously affects the communities and the economy [97].

So far, no approved antiviral therapy or vaccine against CHIKV is on the market, leaving patients with supportive therapy only. This usually consists of analgesics, antipyretics, and fluid therapy. Current recommended therapy for CHIKV-infected patients with arthritis/arthralgia encompass non-steroid anti-inflammatory drugs (NSAIDs) to manage pain and inflammation used along with fluid therapy to prevent dehydration [98]. Aspirin should be avoided, since it bears the risk of bleeding and developing Reye's syndrome [99]. Treatment of CHIKF with corticosteroids might cause immunosuppression and worsen the disease. Furthermore, the adverse effects of corticosteroids make their long-term administration additionally risky, thus causing a problem for the treatment of patients with chronic arthralgia or arthritis.

\section{Prevention and control}

Bite prevention and vector control are the two hallmarks to avoiding infection in the first place. When travelling to endemic areas, general personal protective measures like wearing long-sleeved clothing or using insect repellent and mosquito nets are important to prevent being bitten by a CHIKV positive mosquito. For some vector-borne diseases like Dengue, Chikungunya, Zika, and West Nile disease, vector control is currently the only method available to protect populations (an existing Dengue vaccine is being avoided due to safety concerns [100]).
Before the invention of broad spectrum insecticides in the 1940s, vector control was mainly achieved by environmental management and larval control based on a thorough understanding of pathogen transmission [101]. Vector-control measures can generally be classified into chemical and nonchemical-based approaches. Both strategies can target immature and adult stages either by killing them (with chemical or biological larvicides/adulticides) or by removing the habitat essential for these stages (e.g. the draining of marshes). To reduce contact of the adult vector with the human host, tropical repellents, insecticide treated bed nets or housing improvements are applied [101].

In the past decades, so-called rear and release strategies were brought into focus. In this approach, the intentional mass rearing and release of modified mosquitoes that mate with the wild counterparts aims to induce genetic change, sterility or reduced vector competence into the target population [102]. There have been several programs to achieve this goal. The release of sterile males that if mating with wild females, result in infertile eggs and reduction and eventual elimination of the vector population was one of the first concepts. Males have been sterilised with radiation, chemical methods, the introduction of genetical modification or microbiological methods [103].

Another approach lies in synthetic gene drives. Gene drives are selfish genetic elements that manipulate gametogenesis and reproduction to increase their own transmission to the next generation [104]. Some synthetic gene drives are used to potentially hamper the function of essential genes which hopefully if released, will lead to a decline of the wild population or conversion of the population into males. Other drives aim to modify the vector population in a way that they are more sensitive to pesticides or reduce the vectors' ability to transmit a virus [104].

Aliota et al. [105] followed this strategy of vector control. His research group tried to curb CHIKV transmission by infecting mosquitoes with the intracellular bacterium Wolbachia. The maternally transmitted bacterium Wolbachia is an endosymbiont that manipulates host reproduction to enhance its own transmission [106]. This may result in enhanced resistance to pathogens and reduced virus replication in the vector [107]. Aliota introduced the wMel strain of Wolbachia pipientis into Ae. aegypti mosquitoes and showed that replication and transmission potential of CHIKV were reduced significantly. Similar results have been published for other arboviruses like DENV, Yellow Fever virus and Zika virus, all of which share Ae. aegypty as a common vector [108-110]. It seems that the extent of pathogen reduction can be influenced by the strain of bacterium [110]. In endemic regions thus infected mosquitoes could be released and invade the wild mosquito populations and consequently reduce viral transmission. Studies for curbing Dengue virus transmission by releasing Wolbachia-infected mosquitoes 
into natural Ae. aegypti populations have been started in 2017 seem promising. [111, 112]. According to the World Mosquito Program (WMP) studies conducted in Indonesia over a period of 3 years showed that compared to untreated areas, there is a 77\% reduction in the incidence of virologically confirmed Dengue fever in Wolbachia-treated areas of Yogyakarta, Indonesia (Clinical Trial No. NCT03055585) [113].

If these approaches work out, it might prove an ecofriendly method to curb virus spread without applying chemical insecticides. However, they have their flaws. Rearing and release strategies are expensive, must be well organised, require significant infrastructure, and suffer from an overall negative public opinion, especially when genetically modified mosquito (GMM) are concerned. Furthermore, it is possible, that introduced gene drives evolve back or fail due to drive resistance resulting from standing genetic variation [114].

\section{Prophylaxis and post-exposure therapy}

\section{Active immunisation}

Compared to other RNA viruses, CHIKV displays a limited diversity between the different strains. Various studies showed that antibodies raised by one CHIKV genotype display a cross-reactivity against all others and there is a broad consensus that CHIKV lineages constitute to a single serotype $[28,115]$. This makes CHIKV a viable candidate for generating a vaccine that grants a life-long protection against an infection, with little risk of complications like antibody-dependent enhancement (ADE) as reported from DENV vaccines [116]. Although trying to develop a vaccine since the 1960s, no vaccines have been approved so far, but several candidates are currently being investigated in preclinical and clinical trials [117]. Here, we will only focus on those vaccine candidates that made it to human studies (clinical trials) (Table 2).

Live-attenuated vaccines The advantages of live-attenuated vaccines are that they offer effective and long-lasting immunity, do not have to be given very often and are low in production costs. The first live-attenuated CHIKV vaccine that made it to clinical trials was called TSI-GSD-218 and was developed by successively growing the CHIKV 181/ clone 25 in cell culture [80]. It seemed to provide an effective and lasting immunity. However, in the phase 2 trial $8 \%$ of the vaccinees developed mild arthralgia [81]. The candidate was abandoned after studies indicated instability of attenuation which raised concerns about safety [8, 82]. Yet, the strain is still used as an attenuated lab strain. This candidate revealed a disadvantage of live-attenuated vaccines: the chance of the virus evolving back into an infective strain. In case of TSI-GSD-218, attenuation was determined by two amino acid substitutions in the E2 envelope glycoprotein which seemed not to be very stable $[8,82]$. Research tried to find other strategies for developing live-attenuated vaccines and finally came up with CHIKV/IRES, a candidate that contains an internal ribosomal entry site (IRES) in place of the subgenomic promotor. This IRES leads to a decrease of the expression of viral structural proteins. As a result, replication in mammalian cells is attenuated and replication in mosquito cells is completely prevented because the IRES does not work in insect cells [8]. The CHIKV/IRES vaccine worked in a safe, highly immunogenic, and effective way in studies with mice and NHP $[125,126]$. It furthermore protected mice and NHP against various CHIKV strains and has been preclinically evaluated on safety, efficacy and stability so that now CHIKV/IRES is projected for clinical studies [28, 135].

Another live-attenuated CHIKV vaccine candidate with the number VLA1553 which has been developed by the biotech company Valneva was recently investigated in a phase 1 clinical study to research three dose levels of VLA1553 after a single immunisation (ClinicalTrials.gov; Identifier: NCT03382964). According to the manufacturer, the monovalent, single dose vaccine candidate which was granted Fast Track designation by the FDA in December 2018, had excellent final phase 1 results. Preclinical studies with NHP proved the vaccine candidate to fully protect the animals against WT CHIKV infection after a single dose [118]. Phase 2 supportive studies are ongoing and the candidate has received approval from the FDA to enter phase 3 clinical studies in 2020 [136]. Valneva has recently initiated a pivotal phase 3 trial for the vaccine (NCT NCT04546724). In the randomised, double-blinded, placebo-controlled, multicentre study called VLA1553-301 with approximately 4000 healthy participants, the safety and immunogenicity 28 days after a single-shot vaccination with VLA1553 is to be evaluated. A subset of participants will be tested for sero-protection based on an immunological surrogate (under the Accelerated Approval pathway). Participants will be followed for a total of 6 months [119]. This is the first CHIKV vaccine study to enter phase 3 . The parental strain of VLA1553 is the infectious clone CHIKV LR2006-OPY1 and attenuation was achieved by deleting a major part of the gene encoding for nsP3 [120].

Furthermore, in June 2020 an award of US\$ 14.1 million was awarded by the Coalition for Epidemic Preparedness Innovations (CEPI) for advancing the development of the live-attenuated vaccine candidate BBV87 [121]. The vaccine has been developed by Bharat Biotech (BBIL) and is an inactivated whole virion vaccine based on a strain derived from an East, Central, South African (ECSA) genotype [121]. The vaccine has completed standard preclinical studies, and an optimum immune response was elicited by the 


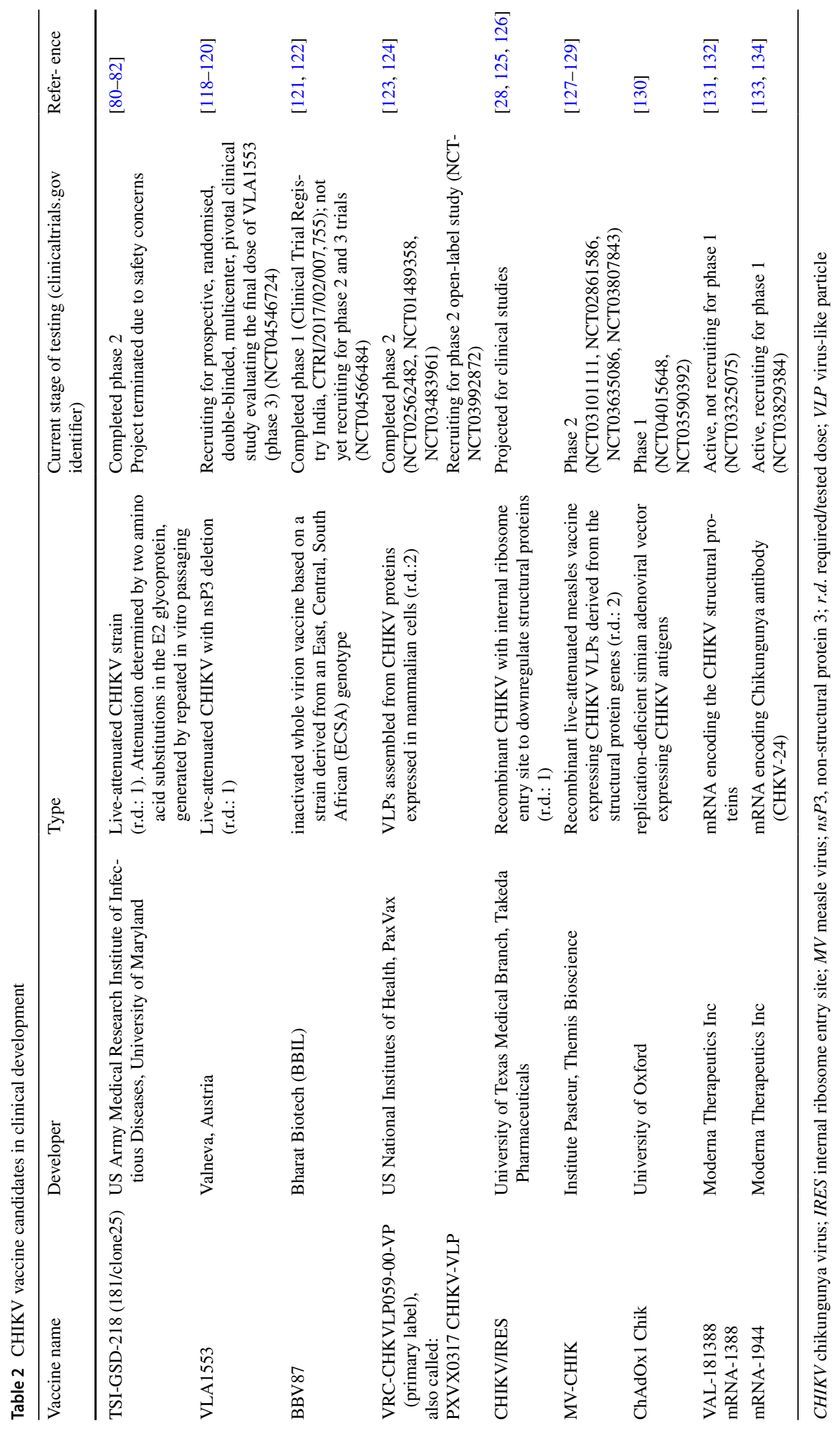


adjuvanted vaccine in phase 1 clinical trials in India (Clinical Trial Registry India, CTRI/2017/02/007755). A phase 2/3 adaptive seamless design, randomised, controlled trial has been initiated in September 2020 to evaluate the safety and immunogenicity of a 2 dose-regimen the mentioned CHIKV vaccine in healthy subjects in Panama, Colombia, and Thailand (NCT04566484). The study is not yet recruiting and further details on the vaccine candidate have not been published yet.

Virus-like particles Another vaccine approach deals with virus-like particles (VLPs) which are generated by transfecting a DNA expression plasmid into human cells. The plasmid encodes for CHIKV structural proteins. After expression these structural proteins form particles that resemble intact virions. Yet, since the virions lack genomic viral RNA, they are unable to replicate [137]. One VLP candidate (referred to as VRC 311) completed preclinical trials with NHP and a phase 1 clinical trial proved the vaccine (now labelled VRC-CHKVLP059-00-VP) to be safe, well tolerated and highly immunogenic with a $100 \%$ seroconversion rate in all dose cohorts after booster immunisations [123]. Furthermore, it displayed a cross-protection against multiple CHIKV strains [124]. The candidate has currently finished phase 2 clinical trials and results are being evaluated (ClinicalTrials.gov; Identifier: NCT02562482).

Just like cells may express a plasmid that encodes for structural proteins, a virus may be recruited as a vector to express the structural proteins of the VLPs. This approach is called viral-vectored vaccines (VVVs).

One VVV candidate called MV-CHIKV was generated by using a recombinant, live-attenuated measles virus (MV) vector that expresses CHIKV VLPs. These VLPs comprise capsid and envelope structural proteins from the CHIKV strain "La Réunion" [127]. This VVV candidate was accepted for a phase 1 clinical trial and it triggered the production of neutralising antibodies in a dose-dependent manner. It also had a seroconversion of $100 \%$ after booster immunisation despite the presence of measles antibodies (which resulted from previous measle vaccinations of some study participants) [128]. The vaccine candidate entered phase 2 clinical trials in 2016 (ClinicalTrials.gov; Identifier: NCT02861586) and the study was completed in 2019. MV-CHIKV turned out to be of good safety, tolerability, and immunogenicity. All treated groups developed neutralising antibodies against CHIKV after one or two immunisations [129]. A study with NHP followed in 2019 in which macaques where challenged with a dose of $1.4 \times 10^{5}$ plaque forming units (PFU) injected subcutaneously 56 days after being vaccinated with MV-CHIKV. None of the previously vaccinated animals showed signs of infection after virus challenge. The developed neutralising antibodies showed a cross-reactivity towards other CHIKV strains. The vaccine candidate proved to be safe, immunogenic, efficacious and worthy of further development towards licensure [138].

Another recombinant VVV candidate (ChAdOx1 Chik) is currently being evaluated in phase 1 clinical trial (ClinicalTrials.gov; Identifier: NCT03590392). ChAdOx1 Chik is a replication-deficient simian adenoviral vector that expresses CHIKV antigens. No results have been published on this project so far [130].

mRNA encoding for structural proteins that will assemble to VLPs Another approach has been launched by a company called Moderna which uses mRNA encoding for CHIKV structural proteins. The idea is to deliver the mRNA into the host cells where it is recognised by ribosomes. Subsequently the proteins which are encoded on the mRNA are produced. In case of the CHIKV vaccine, the mRNA encodes for the structural proteins that will assemble to VLPs once they are translated. The host organism thus recognises the VLPs as foreign, starts an immune response and produces corresponding antibodies [131]. In a phase 1 trial, the safety, tolerability, and immunogenicity of the Chikungunya vaccine candidate called mRNA-1388 in healthy human subjects is currently being investigated (ClinicalTrials.gov; Identifier: NCT03325075) [132].

\section{Passive immunisation with monoclonal antibodies (mAbs)}

Monoclonal antibodies are currently under heavy investigation for their possible use in prophylaxis and post-exposure therapy. So far, none have been licenced for medical use, but some studies seem promising and clinical trials are ongoing [55].

Couderc, Khandoudi [139] demonstrated that isolated anti-CHIKV polyclonal antibodies from patients which were recovering from a CHIKV infection could prevent and treat CHIKV infection in mice. He thus laid the foundation to further investigate antibodies as a means for CHIKV prophylaxis and treatment. In 2014 a clinical trial (clinical trial registration NCT02230163) has been initiated to investigate if transferring anti-CHIKV hyperimmune immunoglobulins that have previously been isolated from CHIKV convalescent donor plasma, may prevent infants with a high risk of mother to child transmission during childbirth from developing a severe form of CHIKF. Although being already initiated in 2014, no results of this study have been published so far. Another phase 1 trial is on its way but not yet recruiting (NCT 04441905) to test the SAR440894 monoclonal antibody (IgG1) directed against the E2 envelope protein of chikungunya virus in a randomised, double-blind study.

Given the fact, that up to all neonatal CHIKV cases in the La Réunion outbreak in 2005/2006 were symptomatic with nearly $20 \%$ resulting in a severe form with involvement of the central nervous system and often leading to permanent 
damage (seizures, cerebral palsy) [55], the outcome of these studies would be of major interest.

mAbs targeting CHIKV surface envelope (E) proteins The CHIKV virion surface has 80 spikes which are built up of trimers of the glycoproteins E1-E3. The role of these surface proteins is to enable receptor-mediated endocytosis and the endosomal fusion which is induced by low $\mathrm{pH}$. E2 forms the top of the spike and seems particularly important to mediate the attachment, binding, and entry of the virus particles. Thus, this E2 surface protein is considered a critical protein at which neutralising human and mouse mAbs could be targeted. Various groups have already identified either mouse or human neutralising mAbs that bind to E1 or E2 [140, 141].

A number of human mAbs have been analysed extensively in vivo and in vitro and some mAbs (C9, 4J21 and $5 \mathrm{M} 16)$ provided full protection against CHIKV viremia and CHIKV-associated arthritis when administered prophylactically or even up to $18 \mathrm{~h} p . i$. in mice [77, 141].

In his study on CHIKV mouse mAbs, Pal, Dowd [94] could show, that combinations of mouse mAbs which were administered prior to CHIKV exposure, protected immunocompromised mice against CHIKV infection and may limit the occurrence of mAb-resistant virus. The latter being of special concern as the viral loads of CHIKV during infection is very high. Furthermore, the humanised $\mathrm{mAb} 152$ provided protection against lethal CHIKV infection in mice and even proved to be highly effective as a post-exposure treatment. Pal took his studies further and investigated mAb no. 152 and 166 in resus macaques. Combination therapy of these mAbs resulted in reduced viral spread and infection in the NHP. However, the mAbs were not able to clear the viral load completely and viral RNA persisted possibly in cell reservoirs that were responsible for actively replicating CHIKV RNA [94].

Broeckel and her group engineered a recombinant human monoclonal antibody (SVIR001) from a human mAb (no. $4 \mathrm{~N} 12$ ) that in previous tests showed prophylactic and postexposure activity against CHIKV infection in mice. The newly developed SVIR001 had the same antigen binding and neutralisation site as the original 4N12. SVIR001 was tested successfully in mice and resus macaques as a post-exposure therapy against CHIKV. The NHP displayed a rapid elimination of viremia in addition to less severe joint infiltration and fewer CHIKF signs than the control group. Broeckel could show that the macaques treated with SVIR001 had a diminished viral burden at both the site of infection as well as at distant sites. Moreover, the activated innate immune cell numbers and pro-inflammatory cytokine and chemokine levels were significantly reduced in the treated animals [96].

Since studies could prove that the B domain of the E2 surface protein is highly conserved across the alphavirus realm, the fact that CHIKV polyclonal antibodies show a crossreactivity and protection against multiple alphaviruses is not surprising [140, 142]. Fox identified 2 mAbs (187 and 265) which are broadly cross-reactive and protected mice against CHIKV, O'nyong-nyong virus (ONNV) and Mayaro virus (MAYV) by blocking viral entry and egress. ONNV shows an $86 \%$ envelope protein amino acid similarity and MAYV a $60 \%$ similarity to CHIKV. Both related viruses may also cause arthritic symptoms like CHIKV [140].

Although mAbs seem viable candidates for CHIKV therapy, it must be noted that the E1 and E2 epitopes on the virion might not always be accessible to therapeutic antibodies due to the dynamic movement of the proteins on the virion surface. This may affect the efficacy of the mAbs. Aside from that CHIKV might find a way to circumvent mAbs binding to the epitopes and create escape mutants. This viral resistance might be limited by using a combination of different mAbs (e.g. cocktails of neutralising mAbs that specifically recognise CHIKV or broadly neutralising mAbs against CHIKV and its closely related alphaviruses) at the same time, thus using synergistic effects to trap these virions before they can interact with the host receptors [143].

The use of mAbs, however, is time sensitive and in the later stages of CHIKF, passive transfer of antibodies usually does not improve the disease outcome. Furthermore, it is questionable whether mAbs can actually reach all cellular places where CHIKV RNA replication occurs. More research is needed as far as kinetics, doses, combinations, and invasiveness of mAbs are concerned. Last but not least, manufacturing mAbs against $\mathrm{CHIKV}$ is not a routine procedure. Cell lines need still to be identified that can effectively produce mAbs according to modern standards. Otherwise, mAb therapy may be too costly and their effective delivery especially to resource-limited areas might be difficult [144].

mRNA encoding for mAbs Another approach for passive immunisation is to deliver mRNA encoding for mAbs into the organism. Kose and colleagues isolated human mAbs from the B cells of a survivor of natural CHIKV infection [133]. Kose created an mRNA sequence that encoded for the mAbs and encapsulated this mRNA into lipid nanoparticles (LNPs). The LNPs were then delivered into mice by infusion. One human mAb, CHIKV-24, was expressed to biologically significant levels in vivo. The group then evaluated the protective capacity of the CHIKV-24 mAb mRNA first in mice, and later in NHP. Treatment with the $\mathrm{mAb}$ encoding mRNA protected mice from typical signs of CHIKV infection like arthritis, musculoskeletal tissue infection and death in a dose-dependent manner. Furthermore, it reduced viremia to undetectable levels at 2 days post inoculation in mice. NHP produced a level of $\mathrm{mAb}$ concentration that was well above the one needed for protection in mice. The NHP showed a dose-response effect after the first dose 
of mRNA and maintained mAb levels after a second dose. Although the NHP were not challenged with WT CHIKV, Klose concluded that the data gathered from his preclinical study suggest that the CHIKV-24 mRNA may be useful to prevent CHIKF in humans [133]. A phase 1 trial is currently taking place to evaluate the safety, tolerability, pharmacokinetics, and pharmacodynamics of mRNA-1944 (ClinicalTrials.gov; Identifier: NCT03829384) [134]. The mRNA is encoding for an anti-CHIKV monoclonal antibody that will be systemically secreted after the mRNA is delivered via infusion into healthy adults.

\section{Challenges in vaccine development and licensure}

The economic and financial burden caused by a CHIKV epidemic are particularly high and the impact of CHIKF in these terms could be eliminated with a safe and effective vaccine [116]. Yet, the road to a licensed vaccine is long and faces multiple challenges.

One bottleneck in the development of a CHIKV vaccine is the fact that substantial funding from private, non-profit, and public institutions is necessary to cover the financial costs that arise until a vaccine is ready for the public. Usually the process costs several hundreds of millions of US dollars and companies need a prospect for a return of their investment, which might be questionable when the highest CHIKV burden occurs in developing countries. The abovementioned focal and sporadic nature of CHIKF outbreaks as well as the establishment of a life-long immunity once the disease has been overcome, are further disincentives to for-profit organisations [116].

Yet, apart from travellers visiting afflicted countries, the military might also have increased interest in a vaccine to protect troops deployed in regions where CHIKV is endemic. Climate change, international travel and other unforeseen factors might promote vector emergence and spread in such a way that even developed countries are at risk of becoming endemic for CHIKV, presenting another potential market for a vaccine. The fact that FDA and the EMA have granted Fast Track- and Priority Medicine status to multiple vaccine candidates should inspire further confidence in the for-profit entities regarding the potential market [145].

Vaccine licensure is regulated by the European Medicines Agency (EMA) and the US Food and Drug Administration (FDA). To receive the approval (a so-called biologics license application (BLA)) for a vaccine (or other biological product) in the USA, the product has to meet the requirements of the Section 351 of the U.S. Public Health Service Act (U.S. Code Title 42-THE PUBLIC HEALTH AND WELFARE). It is eminent to demonstrate that the product is safe, pure and effective and that the facility manufacturing and processing the product meets standards designed to assure that said product stays safe, pure and effective [146]. To prove safety and effectiveness the Code of Federal Regulations states in Title 21, Section $\S 314.126$ how "adequate and well-controlled studies" should be conducted. Trials must be designed in such a way as to distinguish the effect of an investigational product from other influences, such as chance, placebo effect or bias [147]. There are three different approval pathways available under FDA rule and they all require the same level of evidence to prove safety, purity and effectivity. There is the "traditional approval", the "accelerated approval" and the so-called "animal rule" [148].

The "traditional approval" pathway encompasses adequate and well-controlled clinical studies in humans. Recognising the current epidemiological problems and the need for a CHIKV vaccine, the WHO has published a R\&D blueprint in which the principles in the design, conduct and analysis of Phase2b/Phase 3 clinical trials to evaluate Chikungunya vaccines are outlined [149].The WHO's suggested trial design is a Phase 3 prospective, double-blind, placebo-controlled, efficacy trial [149]. While this study design is considered the "gold standard" in epidemiologic studies, it seems that the "traditional approval" pathway does not work to receive a BLA for a CHIKV vaccine for various reasons [145].

The unpredictable, sporadic, focal, and relatively short nature of CHIV outbreaks make classical phase 3 trials impossible, which usually take several months in planning. Finding a suitable trial site months or years in advance is not feasible. The population afflicted mostly with severe disease outcome are the elderly and the very young. Clinical trials must take the immune status of these groups into account and the potential vaccines should have an enhanced safety profile. While live-attenuated vaccines are considered less safe due to their chance of regaining virulence, others, like VLPs are safer but less immunogenic [150]. With regard to a reduced immune system status this could prove problematic. Furthermore, while various studies proved the efficacy of neutralising antibodies to grant protection against CHIKV infection, no defined threshold of neutralising antibodies titres could be defined as a correlate of protection. A lack of standardisation of antibody neutralisation protocols prevents comparison of the different vaccine candidates [149].

The US Code of Federal Regulation (CFR) of the FDA thus allows alternative pathways in licensure for situations in which classical trials are not feasible. In a meeting initiated by the FDA and the Vaccine \& Related Biological Products Advisory Committee (VRBPAC) in November 2019, alternative licensure pathways of CHIKV vaccines were discussed [151]. The consensus of the meeting was that the epidemiology of CHIKV does not allow for classical clinical efficacy trials and that a combination of seroepidemiological studies and non-human primate animal models might be a reasonable way to assess vaccine efficacy [151]. The socalled accelerated approval and the animal rule pathways, which can be used if a disease causes a serious condition, 
are thus legitimate alternatives to the traditional efficacy trials. According to the FDA a serious condition is"a disease or condition associated with morbidity that has substantial impact on day-to-day functioning. Short-lived and self-limiting morbidity will usually not be sufficient, but the morbidity need not be irreversible if it is persistent or recurrent. Whether a disease or condition is serious is a matter of clinical judgement, based on its impact on such factors as survival, day-to-day functioning, or the likelihood that the disease, if left untreated, will progress from a less severe condition to a more serious one" [152]. As CHIKV infection can lead to persistent, disabling polyarthralgia which may last months or even years in up to $50 \%$ of the patients [50], CHIKF does qualify as a serious disease. Both alternative pathways may lead to a BLA without proof of efficacy in human clinical trials. However, clinical efficacy trials in humans still need to be conducted for verification after receiving licensure. Accelerated approval licensure is regulated in 21CFR601 Subpart E. The FDA may grant marketing approval of a biological product if an effect can be demonstrated on a surrogate endpoint that is reasonably likely to predict clinical benefit [153]. The question what might serve as a surrogate endpoint still needs to be answered. It may be possible to use a combination of seroepidemiological studies and non-human primate animal models to create an immunogenic surrogate based on neutralising antibodies [148].

The animal rule pathway only applies if neither "traditional approval" nor accelerated approval is available. Besides other criteria, efficacy of a vaccine may be proven using a sufficiently well-characterised animal model for predicting the response in humans and if animal study endpoint is clearly related to the desired benefit in humans [154]. However, since there are no animal models fit to mimic the chronic state of CHIKV disease (polyarthralgia and the resulting chronic inflammatory erosive arthritis), it is questionable if demonstrating that a CHIKV vaccine grants sterilising immunity against CHIKV in an NHP model suffices to predict benefits in humans.

\section{Conclusion}

With CHIKV transmission depending on arthropod vectors in a complex interaction between virus host and the environment, a thorough understanding of these interactions is essential for the development of strategies to control outbreaks and the geographical spread of vectors. Consideration of factors driving climate change plays an important role, as the vectors might invade habitats that were formerly unsuitable for them.

Countermeasures reviewed here include vector control, prophylaxis, post-exposure therapy or treatment of the disease itself. A number of vaccine candidates look promising and have completed phase 2 clinical trials. Likewise, postexposure therapy with monoclonal antibodies might be a valuable option. However, clinical trials are scarce in this particular field and only one trial is currently recruiting. Past epidemics caused by CHIKV demonstrate the impact a neglected or (re)emerging disease may have on a naïve population. Agents like CHIKV that have the potential to disable a population for a longer period and cause possible long-term sequelae pose a threat to the health and the economic system of a country. In the absence of a licensed vaccine, further research in the area of CHIKV disease prophylaxis is of utmost importance to prevent outbreaks and protect vulnerable populations.

Acknowledgements We thank members of the virology department for critical reading of the manuscript.

\section{References}

1. Powers AM et al (2000) Re-emergence of chikungunya and O'nyong-nyong viruses: evidence for distinct geographical lineages and distant evolutionary relationships. J Gen Virol 81(Pt 2):471-479

2. Brown RS, Wan JJ, Kielian M (2018) The alphavirus exit pathway: what we know and what we wish we knew. Viruses 10(2):89

3. Powers AM et al (2000) Re-emergence of chikungunya and o'nyong-nyong viruses: evidence for distinct geographical lineages and distant evolutionary relationships. J Gen Virol 81(2):471-479

4. Minh BQ et al (2020) IQ-TREE 2: new models and efficient methods for phylogenetic inference in the genomic era. Mol Biol Evol 37(5):1530-1534

5. Kalyaanamoorthy S et al (2017) ModelFinder: fast model selection for accurate phylogenetic estimates. Nat Methods 14(6):587-589

6. Strauss JH, Strauss EG (1994) The alphaviruses: gene expression, replication, and evolution. Microbiol Rev 58(3):491-562

7. Khan AH et al (2002) Complete nucleotide sequence of chikungunya virus and evidence for an internal polyadenylation site. J Gen Virol 83(Pt 12):3075-3084

8. Silva LA, Dermody TS (2017) Chikungunya virus: epidemiology, replication, disease mechanisms, and prospective intervention strategies. J Clin Invest 127(3):737-749

9. Pietila MK, Hellstrom K, Ahola T (2017) Alphavirus polymerase and RNA replication. Virus Res 234:44-57

10. Robinson MC (1955) An epidemic of virus disease in Southern Province, Tanganyika Territory, in 1952-53. I. Clinical features. Trans R Soc Trop Med Hyg 49(1):28-32

11. Kuno G (2015) A re-examination of the history of etiologic confusion between dengue and chikungunya. PLoS Negl Trop Dis 9(11):e0004101

12. Matusali $\mathrm{G}$ et al (2019) Tropism of the chikungunya virus. Viruses 11(2): 175

13. Schuffenecker I et al (2006) Genome microevolution of chikungunya viruses causing the Indian Ocean outbreak. PLoS Med 3(7):e263

14. Althouse BM et al (2018) Role of monkeys in the sylvatic cycle of chikungunya virus in Senegal. Nat Commun 9(1):1046 
15. Tsetsarkin KA et al (2011) Chikungunya virus: evolution and genetic determinants of emergence. Curr Opin Virol $1(4): 310-317$

16. Sam IC et al (2015) Chikungunya virus in macaques, Malaysia. Emerg Infect Dis 21(9):1683-1685

17. Mavale $M$ et al (2010) Venereal transmission of chikungunya virus by Aedes aegypti mosquitoes (Diptera: Culicidae). Am J Trop Med Hyg 83(6):1242-1244

18. Acharya D et al (2015) Loss of glycosaminoglycan receptor binding after mosquito cell passage reduces chikungunya virus infectivity. PLoS Negl Trop Dis 9(10):e0004139

19. Renault $P$ et al (2012) Epidemiology of chikungunya infection on Reunion Island, Mayotte, and neighboring countries. Med Mal Infect 42(3):93-101

20. Yang CF et al (2016) Imported chikungunya virus strains, Taiwan, 2006-2014. Emerg Infect Dis 22(11):1981-1984

21. Angelini R et al (2007) An outbreak of chikungunya fever in the province of Ravenna, Italy. Euro Surveill 12(9):E070906.1

22. Grandadam $\mathrm{M}$ et al (2011) Chikungunya virus, southeastern France. Emerg Infect Dis 17(5):910-913

23. Cauchemez S et al (2014) Local and regional spread of chikungunya fever in the Americas. Euro surveillance: bulletin Europeen sur les maladies transmissibles = European communicable disease bulletin 19(28):20854-20854

24. ECDC (2020) Chikungunya worldwide overview: geographical distribution of chikungunya cases reported worldwide, July 2020 Situation update, 17 July 2020. Available from: https:// www.ecdc.europa.eu/en/chikungunya-monthly

25. Pages F et al (2009) Aedes albopictus mosquito: the main vector of the 2007 chikungunya outbreak in Gabon. PLoS ONE 4(3): 4691

26. Mogi M et al (2017) The climate range expansion of Aedes albopictus (Diptera: Culicidae) in Asia inferred from the distribution of Albopictus subgroup species of Aedes (Stegomyia). J Med Entomol 54(6):1615-1625

27. Sahadeo N et al (2015) Correction: molecular characterisation of chikungunya virus infections in trinidad and comparison of clinical and laboratory features with dengue and other acute febrile cases. PLoS Negl Trop Dis 9(12):e0004305

28. Langsjoen RM et al (2018) Chikungunya virus strains show lineage-specific variations in virulence and cross-protective ability in murine and nonhuman primate models. mBio 9(2):02449-17

29. Tsetsarkin KA et al (2007) A single mutation in chikungunya virus affects vector specificity and epidemic potential. PLoS Pathog 3(12):e201

30. Tsetsarkin KA et al (2009) Epistatic roles of E2 glycoprotein mutations in adaption of chikungunya virus to Aedesalbopictus and Ae. aegypti mosquitoes. PLoS ONE 4(8):e6835-e6835

31. Niyas KP et al (2010) Molecular characterization of chikungunya virus isolates from clinical samples and adult Aedesalbopictus mosquitoes emerged from larvae from Kerala, South India. Virol J 7:189-189

32. Pierro DJ, Powers EL, Olson KE (2008) Genetic determinants of Sindbis virus mosquito infection are associated with a highly conserved alphavirus and flavivirus envelope sequence. J Virol 82(6):2966-2974

33. Tsetsarkin KA, Weaver SC (2011) Sequential adaptive mutations enhance efficient vector switching by chikungunya virus and its epidemic emergence. PLoS Pathog 7(12): $1002412-\mathrm{e} 1002412$

34. Brault AC et al (2004) Venezuelan equine encephalitis emergence: enhanced vector infection from a single amino acid substitution in the envelope glycoprotein. Proc Natl Acad Sci USA 101(31):11344-11349
35. Vashishtha M et al (1998) A single point mutation controls the cholesterol dependence of Semliki Forest virus entry and exit. J Cell Biol 140(1):91-99

36. Sourisseau M et al (2007) Characterization of reemerging chikungunya virus. PLoS Pathog 3(6):e89

37. Wichit $\mathrm{S}$ et al (2017) AedesAegypti saliva enhances chikungunya virus replication in human skin fibroblasts via inhibition of the type I interferon signaling pathway. Infect Genet Evol 55:68-70

38. Zhang $\mathrm{R}$ et al (2018) Mxra8 is a receptor for multiple arthritogenic alphaviruses. Nature 557(7706):570-574

39. Labadie $\mathrm{K}$ et al (2010) Chikungunya disease in nonhuman primates involves long-term viral persistence in macrophages. J Clin Invest 120(3):894-906

40. Ruiz Silva M et al (2016) Mechanism and role of MCP-1 upregulation upon chikungunya virus infection in human peripheral blood mononuclear cells. Sci Rep 6:32288

41. Ozden S et al (2007) Human muscle satellite cells as targets of chikungunya virus infection. PLoS ONE 2(6):e527

42. Couderc T et al (2008) A mouse model for chikungunya: young age and inefficient type-I interferon signaling are risk factors for severe disease. PLoS Pathog 4(2):e29

43. Lokireddy S, Vemula S, Vadde R (2008) Connective tissue metabolism in chikungunya patients. Virol J 5:31

44. Pal P et al (2014) Chikungunya viruses that escape monoclonal antibody therapy are clinically attenuated, stable, and not purified in mosquitoes. J Virol 88(15):8213-8226

45. Mehta R et al (2018) The neurological complications of chikungunya virus: a systematic review. Rev Med Virol 28(3):e1978

46. Cerny $\mathrm{T}$ et al (2017) The range of neurological complications in chikungunya fever. Neurocrit Care 27(3):447-457

47. Das $T$ et al (2015) Multifaceted innate immune responses engaged by astrocytes, microglia and resident dendritic cells against chikungunya neuroinfection. J Gen Virol 96(Pt 2):294-310

48. Inglis FM et al (2016) Neuropathogenesis of chikungunya infection: astrogliosis and innate immune activation. J Neurovirol 22(2): $140-148$

49. Her Z et al (2015) Loss of TLR3 aggravates CHIKV replication and pathology due to an altered virus-specific neutralizing antibody response. EMBO Mol Med 7(1):24-41

50. Rodriguez-Morales AJ et al (2016) Prevalence of post-chikungunya infection chronic inflammatory arthritis: a systematic review and meta-analysis. Arthritis Care Res (Hoboken) 68(12):1849-1858

51. Manimunda SP et al (2010) Clinical progression of chikungunya fever during acute and chronic arthritic stages and the changes in joint morphology as revealed by imaging. Trans R Soc Trop Med Hyg 104(6):392-399

52. Economopoulou A et al (2009) Atypical Chikungunya virus infections: clinical manifestations, mortality and risk factors for severe disease during the 2005-2006 outbreak on Reunion. Epidemiol Infect 137(4):534-541

53. Simon F, Javelle E, Gasque P (2015) Chikungunya virus infections. N Engl J Med 373(1):93-94

54. Renault P, Josseran L, Pierre V (2008) Chikungunya-related fatality rates, Mauritius, India, and Reunion Island. Emerg Infect Dis 14(8): 1327

55. Gerardin P et al (2016) Chikungunya virus-associated encephalitis: a cohort study on La Reunion Island, 2005-2009. Neurology 86(1):94-102

56. Barrera R et al (2011) Preparedness and response for chikungunya virus introduction in the Americas. PAHO/WHO, Washington, DC

57. Omarjee R et al (2014) Importance of case definition to monitor ongoing outbreak of chikungunya virus on a background of 
actively circulating dengue virus, St Martin, December 2013 to January 2014. Euro Surveill 19(13):20753

58. Kaur M et al (2018) Coinfection of chikungunya and dengue viruses: a serological study from North Western region of Punjab, India. J Lab Phys 10(4):443-447

59. Jacobsen $\mathrm{S}$ et al (2016) External quality assessment studies for laboratory performance of molecular and serological diagnosis of chikungunya virus infection. J Clin Virol 76:55-65

60. Johnson BW, Russell BJ, Goodman CH (2016) Laboratory diagnosis of chikungunya virus infections and commercial sources for diagnostic assays. J Infect Dis 214(suppl 5):S471-S474

61. Kikuti M et al (2020) Evaluation of two commercially available chikungunya virus IgM enzyme-linked immunoassays (ELISA) in a setting of concomitant transmission of chikungunya, dengue and Zika viruses. Int J Infect Dis 91:38-43

62. Kam Y-W et al (2015) Sero-prevalence and cross-reactivity of chikungunya virus specific anti-E2EP3 antibodies in arbovirusinfected patients. PLoS Negl Trop Dis 9(1):e3445-e3445

63. Figueiredo LT (2007) Emergent arboviruses in Brazil. Rev Soc Bras Med Trop 40(2):224-229

64. Gaibani P, Landini MP, Sambri V (2016) Diagnostic methods for CHIKV based on serological tools. Methods Mol Biol 1426:63-73

65. Sharp TM et al (2016) Surveillance for chikungunya and dengue during the first year of chikungunya virus circulation in puerto rico. J Infect Dis 214(suppl 5):S475-S481

66. Gobbi F et al (2015) Emergence and surveillance of chikungunya. Curr Trop Med Rep 2(1):4-12

67. Capeding MR et al (2013) Dengue and other common causes of acute febrile illness in Asia: an active surveillance study in children. PLoS Negl Trop Dis 7(7):e2331

68. Roth A et al (2014) Preparedness for threat of chikungunya in the pacific. Emerg Infect Dis 20(8):e130696

69. PPHSN. Pacific Public Health Surveillance Network 2016 [cited 2020 3. October 2020]; Available from: https://www.pphsn.net/ index.htm.

70. Heitmann A et al (2018) Experimental risk assessment for chikungunya virus transmission based on vector competence, distribution and temperature suitability in Europe, 2018. Eurosurveillance 23(29): 1800033

71. ECDC (2017) Cluster of autochtonous chikungunya cases in France-23 August 2017. ECDC-European Centre for Disease Prevention and Control, Stockholm

72. Bugert JJ et al (2020) Antivirals in medical biodefense. Virus Genes 56(2):150-167

73. Patel AC (2013) Clinical relevance of target identity and biology: implications for drug discovery and development. J Biomol Screen 18(10):1164-1185

74. Takenaka T (2001) Classical vs reverse pharmacology in drug discovery. BJU Int 88(Suppl 2):7-10

75. Rester U (2008) From virtuality to reality_virtual screening in lead discovery and lead optimization: a medicinal chemistry perspective. Curr Opin Drug Discov Devel 11(4):559-568

76. Pohjala L et al (2011) Inhibitors of alphavirus entry and replication identified with a stable chikungunya replicon cell line and virus-based assays. PLoS ONE 6(12):e28923

77. Selvarajah $\mathrm{S}$ et al (2013) A neutralizing monoclonal antibody targeting the acid-sensitive region in chikungunya virus $\mathrm{E} 2$ protects from disease. PLoS Negl Trop Dis 7(9):e2423

78. Weber $\mathrm{C}$ et al (2015) The green tea catechin, epigallocatechin gallate inhibits chikungunya virus infection. Antiviral Res 113:1-3

79. Lucas-Hourani M et al (2013) A phenotypic assay to identify chikungunya virus inhibitors targeting the nonstructural protein nsP2. J Biomol Screen 18(2):172-179
80. Levitt NH et al (1986) Development of an attenuated strain of chikungunya virus for use in vaccine production. Vaccine 4(3): 157-162

81. Edelman R et al (2000) Phase II safety and immunogenicity study of live chikungunya virus vaccine TSI-GSD-218. Am J Trop Med Hyg 62(6):681-685

82. Gorchakov R et al (2012) Attenuation of chikungunya virus vaccine strain $181 /$ clone 25 is determined by two amino acid substitutions in the E2 envelope glycoprotein. J Virol 86(11):6084-6096

83. Khan $\mathrm{M}$ et al (2011) Cellular IMPDH enzyme activity is a potential target for the inhibition of chikungunya virus replication and virus induced apoptosis in cultured mammalian cells. Antiviral Res 89(1):1-8

84. Rathore AP et al (2014) Chikungunya virus nsP3 \& nsP4 interacts with HSP-90 to promote virus replication: HSP-90 inhibitors reduce $\mathrm{CHIKV}$ infection and inflammation in vivo. Antiviral Res 103:7-16

85. Briolant $S$ et al (2004) In vitro inhibition of chikungunya and Semliki Forest viruses replication by antiviral compounds: synergistic effect of interferon-alpha and ribavirin combination. Antiviral Res 61(2):111-117

86. Delang L et al (2014) Mutations in the chikungunya virus non-structural proteins cause resistance to favipiravir (T-705), a broad-spectrum antiviral. J Antimicrob Chemother 69(10):2770-2784

87. Cruz DJ et al (2013) Identification of novel compounds inhibiting chikungunya virus-induced cell death by high throughput screening of a kinase inhibitor library. PLoS Negl Trop Dis 7(10):e2471

88. Bassetto $\mathrm{M}$ et al (2013) Computer-aided identification, design and synthesis of a novel series of compounds with selective antiviral activity against chikungunya virus. Antiviral Res 98(1):12-18

89. Bourjot $\mathrm{M}$ et al (2014) Trigocherrierin A, a potent inhibitor of chikungunya virus replication. Molecules 19(3):3617-3627

90. Jadav SS et al (2015) Thiazolidone derivatives as inhibitors of chikungunya virus. Eur J Med Chem 89:172-178

91. Teng TS et al (2012) Viperin restricts chikungunya virus replication and pathology. J Clin Invest 122(12):4447-4460

92. Abdelnabi $\mathrm{R}$ et al (2017) Protein kinases $\mathrm{C}$ as potential host targets for the inhibition of chikungunya virus replication. Antiviral Res 139:79-87

93. Marlina S et al (2015) Development of a Real-Time Cell Analysing (RTCA) method as a fast and accurate screen for the selection of chikungunya virus replication inhibitors. Parasit Vectors 8:579

94. Pal P et al (2013) Development of a highly protective combination monoclonal antibody therapy against chikungunya virus. PLoS Pathog 9(4):e1003312

95. Parashar D et al (2013) Administration of E2 and NS1 siRNAs inhibit chikungunya virus replication in vitro and protects mice infected with the virus. PLoS Negl Trop Dis 7(9):e2405

96. Broeckel $\mathrm{R}$ et al (2017) Therapeutic administration of a recombinant human monoclonal antibody reduces the severity of chikungunya virus disease in rhesus macaques. PLoS Negl Trop Dis 11(6):e0005637

97. Feldstein LR et al (2019) Estimating the cost of illness and burden of disease associated with the 2014-2015 chikungunya outbreak in the U.S. Virgin Islands. PLoS Negl Trop Dis 13(7):e0007563

98. CDC. Chikungunya Virus. 2020 19. Sept. 2019 [cited 2020]; Available from: https://www.cdc.gov/chikungunya/geo/index .html

99. Kucharz EJ, Cebula-Byrska I (2012) Chikungunya fever. Eur J Intern Med 23(4):325-329

100. Sridhar $\mathrm{S}$ et al (2018) Effect of dengue serostatus on dengue vaccine safety and efficacy. N Engl J Med 379(4):327-340 
101. Wilson AL et al (2020) The importance of vector control for the control and elimination of vector-borne diseases. PLoS Negl Trop Dis 14(1):e0007831-e0007831

102. Ritchie SA, Johnson BJ (2017) Advances in vector control science: rear-and-release strategies show promise ... but don't forget the basics. J Infect Dis 215(suppl_2):S103-S108

103. Alphey L et al (2010) Sterile-insect methods for control of mosquito-borne diseases: an analysis. Vector Borne Zoonotic Dis 10(3):295-311

104. Wedell N, Price TAR, Lindholm AK (1917) Gene drive: progress and prospects. Proc Biol Sci 2019(286):20192709-20192709

105. Aliota MT et al (2016) The wMel strain of Wolbachia reduces transmission of chikungunya virus in Aedesaegypti. PLoS Negl Trop Dis 10(4):e0004677

106. Werren JH, Baldo L, Clark ME (2008) Wolbachia: master manipulators of invertebrate biology. Nat Rev Microbiol 6(10):741-751

107. Johnson KN (2015) The impact of Wolbachia on virus infection in mosquitoes. Viruses 7(11):5705-5717

108. Aliota MT et al (2016) The wMel strain of Wolbachia reduces transmission of zika virus by Aedes aegypti. Sci Rep 6:28792

109. Hoffmann AA et al (2011) Successful establishment of Wolbachia in Aedes populations to suppress dengue transmission. Nature 476(7361):454-457

110. van den Hurk AF et al (2012) Impact of Wolbachia on infection with chikungunya and yellow fever viruses in the mosquito vector Aedesaegypti. PLoS Negl Trop Dis 6(11):e1892

111. Callaway E (2020) The mosquito strategy that could eliminate dengue. Nature. https://doi.org/10.1038/d41586-020-02492-1

112. Ryan P et al (2020) Establishment of wMel Wolbachia in Aedes aegypti mosquitoes and reduction of local dengue transmission in Cairns and surrounding locations in northern Queensland, Australia [version 2; peer review: 2 approved]. Gates Open Res 3:1547

113. Anders KL et al (2020) Update to the AWED (Applying Wolbachia to Eliminate Dengue) trial study protocol: a cluster randomised controlled trial in Yogyakarta, Indonesia. Trials 21(1):429

114. Callaway E (2017) Gene drives thwarted by emergence of resistant organisms. Nature 542(7639):15

115. Nitatpattana $\mathrm{N}$ et al (2014) Long-term persistence of chikungunya virus neutralizing antibodies in human populations of North Eastern Thailand. Virol J 11:183

116. Rezza G, Weaver SC (2019) Chikungunya as a paradigm for emerging viral diseases: evaluating disease impact and hurdles to vaccine development. PLOS Negl Trop Dis 13(1):e0006919

117. Reyes-Sandoval A (2019) 51 years in of chikungunya clinical vaccine development: a historical perspective. Hum Vaccin Immunother 15(10):2351-2358

118. Roques $\mathrm{P}$ et al (2017) Attenuated and vectored vaccines protect nonhuman primates against chikungunya virus. JCI Insight 2(6):e83527

119. Valneva (2020) Valneva initiates phase 3 clinical study for its chikungunya vaccine candidate VLA1553. Valneva SE, Saint-Herblain

120. Hallengard D et al (2014) Novel attenuated chikungunya vaccine candidates elicit protective immunity in C57BL/6 mice. J Virol 88(5):2858-2866

121. International Vaccine Institute, I. (2020) CEPI awards up to US\$14.1million to consortium of IVI and Bharat Biotech to advance development of Chikungunya vaccine in collaboration with Ind-CEPI. CEPI, the Coalition for Epidemic Preparedness Innovations, Oslo, Seoul, Telangana

122. Clinical Trials Registry-India, C. (2017) Phase-I open label, dose-escalation clinical trial to evaluate the safety, tolerability and immunogenicity of chikungunya vaccine in healthy adults of 18 to 50 years age. Clinical Trials Registry-India, CTRI, Hyderabad

123. Chang LJ et al (2014) Safety and tolerability of chikungunya virus-like particle vaccine in healthy adults: a phase 1 doseescalation trial. Lancet 384(9959):2046-2052

124. Goo L et al (2016) A virus-like particle vaccine elicits broad neutralizing antibody responses in humans to all chikungunya virus genotypes. J Infect Dis 214(10):1487-1491

125. Plante $\mathrm{K}$ et al (2011) Novel chikungunya vaccine candidate with an IRES-based attenuation and host range alteration mechanism. PLoS Pathog 7(7):e1002142

126. Roy CJ et al (2014) Chikungunya vaccine candidate is highly attenuated and protects nonhuman primates against telemetrically monitored disease following a single dose. J Infect Dis 209(12):1891-1899

127. Brandler $\mathrm{S}$ et al (2013) A recombinant measles vaccine expressing chikungunya virus-like particles is strongly immunogenic and protects mice from lethal challenge with chikungunya virus. Vaccine 31(36):3718-3725

128. Ramsauer $\mathrm{K}$ et al (2015) Immunogenicity, safety, and tolerability of a recombinant measles-virus-based chikungunya vaccine: a randomised, double-blind, placebo-controlled, active-comparator, first-in-man trial. Lancet Infect Dis 15(5):519-527

129. Reisinger EC et al (2019) Immunogenicity, safety, and tolerability of the measles-vectored chikungunya virus vaccine MVCHIK: a double-blind, randomised, placebo-controlled and active-controlled phase 2 trial. Lancet 392(10165):2718-2727

130. Medicine, N.N.L.o. ClinicalTrials.gov. [cited 2020 05.05.2020]; Available from: https://clinicaltrials.gov/ct2/show/study/NCT03 590392

131. Moderna. Infectious Diseases. 2019 [cited 2020 January 15]; Available from: https://www.modernatx.com/pipeline/therapeuti c-areas/infectious-diseases

132. Liu, A. Moderna initiates Chikungunya vaccine human test, replaces backup Zika candidate. 2017 [cited 2019 September 19]; Available from: https://www.fiercepharma.com/vaccines/ moderna-initiates-chikungunya-vaccine-human-test-replacesbackup-zika-candidate

133. Kose $\mathrm{N}$ et al (2019) A lipid-encapsulated mRNA encoding a potently neutralizing human monoclonal antibody protects against chikungunya infection. Sci Immunol. https://doi. org/10.1126/sciimmunol.aaw6647

134. Moderna (2019) Moderna announces positive phase 1 results for the first systemic messenger RNA therapeutic encoding a secreted protein (mRNA-1944). Business wire, Cambridge

135. Plante KS et al (2015) Extended preclinical safety, efficacy and stability testing of a live-attenuated chikungunya vaccine candidate. PLoS Negl Trop Dis 9(9):e004007

136. Valneva Press Release (2019) Valneva reports excellent final phase 1 results for its chikungunya vaccine candidate, confirms plans. Valneva, Saint-Herblain

137. Akahata $\mathrm{W}$ et al (2010) A virus-like particle vaccine for epidemic chikungunya virus protects nonhuman primates against infection. Nat Med 16(3):334-338

138. Rossi SL et al (2019) Immunogenicity and efficacy of a measles virus-vectored chikungunya vaccine in nonhuman primates. $\mathrm{J}$ Infect Dis 220(5):735-742

139. Couderc $T$ et al (2009) Prophylaxis and therapy for chikungunya virus infection. J Infect Dis 200(4):516-523

140. Fox JM et al (2015) Broadly neutralizing alphavirus antibodies bind an epitope on E2 and inhibit entry and egress. Cell 163(5):1095-1107

141. Smith SA et al (2015) Isolation and characterization of broad and ultrapotent human monoclonal antibodies with therapeutic activity against chikungunya virus. Cell Host Microbe 18(1):86-95 
142. Kielian M, Saphire EO (2015) Potent antibody protection against an emerging alphavirus threat. Cell 163(5):1053-1054

143. Kuhn RJ et al (2015) Shake, rattle, and roll: impact of the dynamics of flavivirus particles on their interactions with the host. Virology 479-480:508-517

144. Clayton AM (2016) Monoclonal antibodies as prophylactic and therapeutic agents against chikungunya virus. J Infect Dis 214(suppl 5):S506-s509

145. Schrauf $S$ et al (2020) Current efforts in the development of vaccines for the prevention of zika and chikungunya virus infections. Front Immunol 11:592

146. FDA, F.a.D.A. (2018) United States Code, 2018 Edition, Title 42-The public health and welfare. United States Code. U.S. Government Publishing Office, Washington DC

147. FDA, F.a.D.A. (2020) CFR-Title 21-Food and Drugs, Section $\S 314.126$ - adequate and well-controlled studies. In: U.S.D.o.H.a.H.S. (HHS) (ed) Code of Federal Regulations (CFR). Food and Drug Administration (FDA), Washington DC

148. Yang $S$ et al (2017) Regulatory considerations in development of vaccines to prevent disease caused by chikungunya virus. Vaccine 35(37):4851-4858

149. WHO (2018) WHO consultation on Chikungunya vaccine evaluation, in R\&D blueprint. World Health Organisation (WHO), Geneva

150. DeFilippis VR (2019) Chikungunya virus vaccines: platforms, progress, and challenges. Curr Top Microbiol Immunol. https:// doi.org/10.1007/82_2019_175
151. VRBPAC, V.R.B.P.A.C (2018) Vaccines and Related Biological Products Advisory Committee November 8, 2019. FDA US Food \& Drug Administration, Silver Spring

152. FDA, F.a.D.A. (2020) CFR-Title 21-Food and Drugs, Section $\S 312.300$ General. In: U.S.D.o.H.a.H.S. (HHS) (ed) Code of Federal Regulations (CFR). Food and Drug Administration (FDA), Washington DC

153. FDA, F.a.D.A. (2020) CFR—Title 21-Food and Drugs, Section $\S 601.41$ Approval based on a surrogate endpoint or on an effect on a clinical endpoint other than survival or irreversible morbidity. In: U.S.D.o.H.a.H.S. (HHS) (ed) Code of Federal Regulations (CFR). Food and Drug Administration (FDA), Washington DC

154. FDA, F.a.D.A. (2020) CFR - Title 21 - Food and Drugs, Section $\S 601.91$ Approval based on evidence of effectiveness from studies in animals. In: U.S.D.o.H.a.H.S. (HHS) (ed) Code of Federal Regulations (CFR). Food and Drug Administration (FDA), Washington DC

Publisher's Note Springer Nature remains neutral with regard to jurisdictional claims in published maps and institutional affiliations. 